\title{
Research on the Interior Sound Quality in Hybrid Electric Vehicle
}

\author{
Lian Ying LIAO ${ }^{1,2, a}$, Yan Yan ZUO ${ }^{1}$ and Hao Dong MENG ${ }^{2}$ \\ ${ }^{1}$ Institute of Noise and Vibration, Jiangsu University, Zhenjiang, 212013, China \\ ${ }^{2}$ Changzhou Institute of Technology, Changzhou, 213002, China
}

\begin{abstract}
Even the overall level of vehicle interior noise of hybrid electric vehicle (HEV) reduced to a certain degree, the vibration and noise generated by the engine, motor, generator and power split have made greater effect on the vehicle interior sound quality in HEV. In order to research the feature of vehicle interior sound quality in HEV, the HEV is used to be the research object, the binaural noise sample of the driver when playing different kinds of music in the vehicle with the speed of sixty kilometers per hour is collected. ArtemiS is used to conduct frequency division processing, so as to obtain the relative weight of each frequency band and the overall noise. The tone, roughness and sharpness of sound quality subjective evaluation parameters are quantified, the SPSS is used to establish the linear regression model of the sample, and the best masking music tracks are found out. Then, the sound samples that contains the best music tracks and the simple vehicle interior noise are re-collected, the regression model and ArtemiS are used to predict the subjective evaluation value. The research results show that when adding the music, the tone degree rises and the lowering degree decreases, thus the disturbing degree reduces, which significantly improves the sound quality in the HEV.
\end{abstract}

\section{Introduction}

The strength of music in vehicle is usually higher than the noise level. At the same time, music spectrum is completely different with the low frequency noise in vehicle. Besides, tone is also an important characteristic. These factors can effectively distribute the attention of people on the low frequency noise in vehicle, so as to improve the vehicle interior sound quality $[1,2,3]$. In order to analyze the improvement degree of music on the vehicle interior sound quality, 24 tracks that are selected in accordance with the provisions are played in vehicle with the uniform speed condition of $60 \mathrm{~km} / \mathrm{h}$ of HEV [4], and the 24 sound samples contain tracks and vehicle interior low frequency noise collected by SQuadriga I and binaural microphone are studied. Finally, combined with the subjective evaluation criteria and psychoacoustic acoustics parameters, the masking effect of music is subjectively analyzed and predicted. The study results provide theoretical guidance for improving the vehicle interior sound quality in HEV.

\section{The subjective evaluation model of sound quality}

The masking effect of music means that uses the method of playing music in vehicle to weaken the interference of low frequency noise in vehicle on ears, so as to achieve the purpose of improving the vehicle interior sound quality. At present, the subjective

\footnotetext{
$\overline{{ }^{a} \text { Corresponding author: liaolianying } @ 163 . c o m}$
}

evaluation method is usually used to measure the improvement degree of vehicle interior sound quality. Since the final evaluation index of sound quality is the subjective feeling of people, thus the psychoacoustic acoustics parameters, such as language articulation, tone, loudness, sharpness, roughness ect. are usually used to assist the subjective evaluation and analysis.

In the music, there are many single-tone components. The tone is just the main description on the proportion of single frequency component in noise. Its calculation model is shown in formula (1).

$$
K=C \cdot W_{N}^{0.79} \cdot\left(\sqrt{\sum_{i=1}^{N}\left[W_{1}\left(\Delta z_{i}\right) W_{2}\left(f_{i}\right) W_{3}\left(\Delta L_{i}\right)\right]^{2}}\right)^{0.29}
$$

In the formula, $\mathrm{C}$ is the normalized constant; $\mathrm{WN}$ means the relationship between loudness of no-single frequency component $\mathrm{NN}$ and loudness of single frequency component $\mathrm{N} ; \mathrm{W} 1(\Delta \mathrm{zi})$ means the relationship between ith single frequency component and the critical frequency band difference; W2(fi) means the relationship between ith single frequency component and the frequency; $\mathrm{W} 3(\Delta \mathrm{Li})$ means the sound level surplus effect of ith single frequency component. the values are as formula (2), formula (3) , formula (4) and formula (5).

$$
\begin{aligned}
W_{N} & =1-N_{N} / N \\
W_{1}\left(\Delta z_{i}\right) & =[0.13 /(\Delta z+0.13)]^{1 / 0.29}
\end{aligned}
$$




$$
\begin{gathered}
W_{2}\left(f_{i}\right)=1 / \sqrt{1+0.2\left(f_{i} / 700+700 / f_{i}\right)^{2}} \\
W_{3}\left(\Delta L_{i}\right)=\left(1-e^{-\Delta L_{i} / 15}\right)
\end{gathered}
$$

The loudness difference value of left and right ear in high frequency band is very large, which needs to be corrected, so as to correctly describe the subjective feelings in ears [5], the correction model is shown in formula (6).

$$
N_{E Q}=N_{M}+0.04 \times\left(e^{I L D / 4}-1\right)
$$

In the formula, $N_{E Q}$ is the perception value of loudness in ears; $N_{M}$ is the arithmetic average value of loudness in ears; ILD is the sound pressure level difference in ears.

The roughness describes the change relationship between loudness and time in the range of $20 \sim 300 \mathrm{~Hz}$ of modulation frequency, which gives people a kind of rough psychological feeling, the roughness after music masking is shown in formula (7).

$$
R=0.3 f_{\bmod } \int_{0}^{24 B a r k} \Delta L d z
$$

In the formula, fmod is the modulation frequency; $\Delta L$ is the masking depth.

When the modulation frequency is $0.25 \sim 20 \mathrm{~Hz}$, the change of loudness with time is more slowly, which gives people a kind of jitter feeling, that is fluctuation, the value is shown in formula (8).

$$
F=0.36 \int_{0}^{24 B a r k} \log \left(N_{\max }^{\prime} / N_{\min }^{\prime}\right) d z /(T / 0.25+0.25 / T)
$$

In the formula, $N^{\prime}{ }_{\max }$ and $N^{\prime}{ }_{\text {min }}$ are the greatest feature loudness and the smallest feature loudness; $T$ means the short time difference of two consecutive loudness.

Since the evaluation personnel does not have professional training in advance, at the same time, each sample may only has subtle difference, therefore, the paired comparison method is selected to be used as the subjective evaluation criterion [6]. Make ranking of $\mathrm{N}$ samples with two in a group, there are N! kinds of combinations in total. Make comparison playing and let the evaluation team select relatively sweet samples, finally, make quantization process on the results, as shown in table 1 .

Table 1. Subjective evaluation test table of paired comparison method.

\begin{tabular}{|c|c|c|c|}
\hline Group & $\begin{array}{c}\mathrm{A} \text { is sweeter } \\
\text { than B }\end{array}$ & $\begin{array}{c}\mathrm{A} \text { is as } \\
\text { sweet as B }\end{array}$ & $\begin{array}{c}\mathrm{B} \text { is sweeter } \\
\text { than } \mathrm{A}\end{array}$ \\
\hline$N_{1}$ & & & \\
\hline$N_{2}$ & & & \\
\hline$\ldots$ & & & \\
\hline
\end{tabular}

As shown in table 1 , in the same group, if $\mathrm{A}$ is sweeter than B, A gets 2 scores, $\mathrm{B}$ gets 0 score. If $\mathrm{A}$ is as sweet as B, A and B get 1 score. If $\mathrm{B}$ is sweeter than A, B gets 2 scores. When the evaluation is completed, make the unity and normalize the results [7].

\section{Collection and pretreatment of sound samples}

The driver wears binaural microphone, vice-driver holds HEAD portable sound analyzer SQuadriga I. According to GB/T18697, selecting the high speed road with few vehicles and the sampling frequency is $44.1 \mathrm{kHz}$, the sound signals of 26 music with different styles played in vehicle with the speed of $60 \mathrm{~km} / \mathrm{h}$ are collected. Through the subjective comparison, remove 2 acoustic signals that are seriously interfered, and then get 24 sound samples with vehicle interior low frequency noise.

\subsection{Equal loudness process}

The collected sound samples are concentrated in the range of $40 \mathrm{~dB} \sim 60 \mathrm{~dB}$, different loudness makes great effect on the subjective feeling of people. Refer to the sound sample with the loudness value of 20song, make equal loudness process on 24 sound samples. Table 2 lists the change circumstances of all the parameters of sound sample 2 and sound sample 6 before and after equal loudness process.

Table 2. All the parameters of sample 2 and sample 6 before and after equal loudness process.

\begin{tabular}{|c|c|c|c|c|}
\hline $\begin{array}{c}\text { Objective } \\
\text { parameters }\end{array}$ & $2 \#$ & $\begin{array}{c}2 \# \\
\text { amendment }\end{array}$ & $6 \#$ & $\begin{array}{c}6 \# \\
\text { amendment }\end{array}$ \\
\hline Loudness & 17.8 & 19.7 & 25.95 & 20.15 \\
\hline Sharpness & 1.08 & 1.115 & 2.035 & 1.895 \\
\hline Roughness & 1.735 & 1.84 & 1.715 & 1.495 \\
\hline Fluctuation & 0.047 & 0.049 & 0.059 & 0.053 \\
\hline Tone & 0.079 & 0.078 & 0.266 & 0.274 \\
\hline $\begin{array}{c}\text { A sound } \\
\text { pressure } \\
\text { level }\end{array}$ & 62.9 & 64.4 & 66.75 & 62.95 \\
\hline
\end{tabular}

Before the amendment, N2\#<20song, N6\#>20song, after the amendment, the values are about 20song. This can effectively avoid the subjective evaluation error caused by the loudness. Through the comparison, the sharpness, roughness, jitter degree, tone and other parameters basically maintain the positive relationship with loudness. In essence, equal loudness process is equivalent to a weight function. It does not change the differences relationship between all the parameters while amending the loudness.

\subsection{Filtering in different frequency band}

In order to study the effect of different frequency bands on the overall sound quality, at the same time considering too much number of samples will cause the fatigue of evaluators, so as to effect the accuracy of the results, divide the sound samples into 4 frequency bands: mid-low frequency $(20 \sim 160 \mathrm{~Hz})$, mid frequency $(160 \sim 1280 \mathrm{~Hz})$, mid-high frequency $(1280 \sim 4000 \mathrm{~Hz})$ and high frequency (higher than $4000 \mathrm{~Hz}$ ). Conduct division 
frequency band filtering on the 24 samples after equal loudness process and then get 96 division frequency band sample signals.

\section{Subjective evaluation analysis on sound quality}

\subsection{Subjective evaluation process}

As for most of the psychological evaluation tests, 20 evaluators can get more accurate results [8]. 24 students and teachers that have driving experience and are more familiar with the vehicle interior noise environment are selected as the main evaluators. The proportion of men and women in evaluators is selected as $3: 1$. Before the subjective evaluation, conduct sound listening training on the evaluators, so as to unify the evaluation standard of subjective evaluation.

Sum up all the evaluation scores of each evaluators on each sound sample, thus get the preference evaluation value of each evaluators on the sound sample. Use SPSS software to conduct the correlation analysis on the evaluation result of each evaluators and the evaluation results of other evaluators. After the analysis, remove the evaluation results whose correlation coefficients are less than 0.7. Finally, conduct normalization on the evaluation result according to formula (9).

$$
X=0.8\left(X_{i}-X_{\min }\right) /\left(X_{\max }-X_{\min }\right)+0.1
$$

$X_{i}$ is the random evaluation score; $X_{\min }$ and $X_{\max }$ are the minimum value and maximum value in the same group. The scatter diagram and linear relationship in the whole frequency band and division frequency bands are shown in Fig.1.
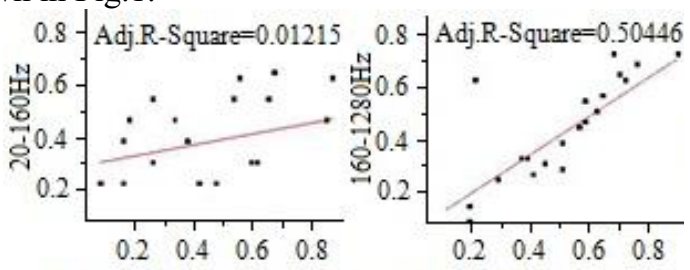

The whole frequency band
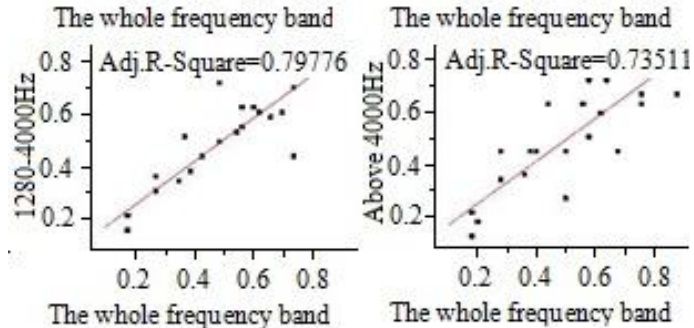

Fig.1. Scatter diagram and linear relationship

Fig. 1 shows that, the adjustment determination coefficients Adj.R-Square of the whole frequency band, mid-low frequency band, mid frequency band, mid-high frequency band and high frequency band are 0.01215, $0.50446,0.79779$ and 0.73511 . The larger the Adj.RSquare is, the determination coefficient $\mathrm{R}$ is larger, and the correlation degree is higher. Therefore, the effect of $1280 \sim 4000 \mathrm{~Hz}$ frequency band on the subjective evaluation of the whole frequency band is the largest. That frequency band mainly reflects the tone of samples. Secondly, the relationship of high frequency band with more than $4000 \mathrm{~Hz}$ is most closed to the language articulation and intelligibility. But if the frequency band has too many ingredients, the sharpness will be too large, which generates auditory fatigue on people. Low frequency samples are mainly come from the vehicle interior low frequency noise, while the music has very little low frequency component. Therefore, the auditory feelings of $20 \sim 160 \mathrm{~Hz}$ are almost the same, which have smallest effect on the whole frequency band. In the following linear regression model, the sound samples of $20 \sim 160 \mathrm{~Hz}$ are neglected.

\subsection{Subjective evaluation and linear regression analysis}

Assume $y_{k}$ is the subjective evaluation value of samples, among them, $k=1,2,3,4$ separately represent the entire frequency band, $160 \sim 1280 \mathrm{~Hz}, 1280 \sim 4000 \mathrm{~Hz}$ and more than $4000 \mathrm{~Hz}$ frequency band. $x_{k i}$ represents the subjective evaluation parameter of $k$ frequency band. Among them, $i=1 \sim 8$ separately represent the loudness, sharpness, roughness, fluctuation, tone, AI index, A sound pressure level, linear sound pressure level of $k$ frequency band. The subjective evaluation models of the four frequency bands are expressed and shown in formula (10).

$$
[y]=[\beta] \cdot[x]+[\varepsilon]
$$

In the formula, $[y]$ is the subjective evaluation value matrix, $[\beta]$ is the regression coefficient matrix, $[x]$ is the subjective parameter matrix, $[\varepsilon]$ is the residual matrix.

Use SPSS software to establish the multiple linear regression model of samples, the fitting degree inspection is shown in table 3 .

Table 3. Fitting degree inspection of multiple linear regression model.

\begin{tabular}{|l|c|c|c|}
\hline \multicolumn{1}{|c|}{ Model } & $R$ & Error $\varepsilon$ & $\begin{array}{c}\text { Durbin- } \\
\text { Watson }\end{array}$ \\
\hline $\begin{array}{l}\text { The whole } \\
\text { frequency band }\end{array}$ & 0.863 & 0.1385 & 2.157 \\
\hline $160 \sim 1280 \mathrm{~Hz}$ & 0.932 & 0.1031 & 1.768 \\
\hline $1280 \sim 4000 \mathrm{~Hz}$ & 0.906 & 0.1103 & 1.419 \\
\hline Over $4000 \mathrm{~Hz}$ & 0.889 & 0.13996 & 2.366 \\
\hline
\end{tabular}

The $\mathrm{R}$ value of each frequency band is very close to 1 and the fitting degree is very good. At the same time, the statistic of Durbin-Watson is about 2, which shows the residual error obeys the normal distribution. Using the method of linear regression analysis to establish the subjective evaluation model is effective. The regression coefficient matrix and residual matrix are shown in formula (11) and formula (12).

$[\boldsymbol{\beta}]=\left[\begin{array}{cccccccc}0.089 & -0.048 & 1.283 & 16.075 & 5.012 & -1.531 & -0.09 & 0.089 \\ -0.033 & 2.331 & -1.503 & 11.818 & 0.322 & -0.593 & -0.149 & 0.207 \\ -0.006 & 0.084 & -0.585 & 13.127 & 0.438 & 3.176 & 0.126 & -0.076 \\ 0.191 & -0.044 & 0.614 & -2.21 & 1.058 & -3.494 & -0.158 & 0.125\end{array}\right]$

$$
[\varepsilon]=\{-5.574,-4.171,-5.171,4.74\}^{T}
$$




\section{The effect of music on vehicle interior sound quality}

Through the subjective evaluation analysis before, the preference character of sample 20 and sample 23 are found to be good, while the subjective evaluation result of sample 12 is worst. Therefore, sample 20 and sample 23 are more suitable to mask the low frequency noise in the vehicle. In order to quantify the effect of music masking effect on vehicle interior noise, re-collect the vehicle interior noise sample of No. 20 and the simple vehicle interior noise sample according to the previous acquisition process. After the process of HEAD ArtemiS, the differences of the two samples in tone, roughness and sharpness are found to be the largest. The results are shown in Fig.2.
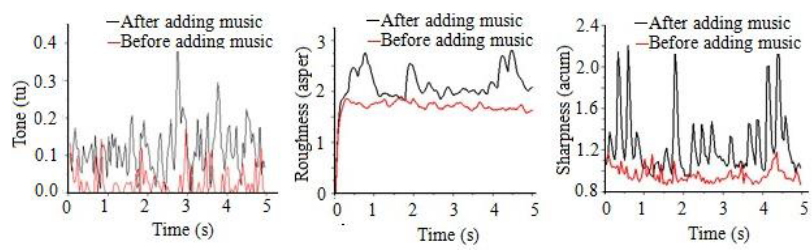

Fig.2. Subjective evaluation parameters change before and after adding music

The tone, roughness and sharpness in the original low frequency noise in vehicle are milder, which is a kind of more low-pitched acoustic environment. In general, when staying in this kind of environment for a long time, people will feel more oppressive and the attention is easily to be distracted, which effects the driving safety. After adding the music, the tone, roughness and sharpness of samples rise obviously. After the sense of rhythm in vehicle being strengthened, the fatigue feelings generated in low-pitched acoustic environment in vehicle can be avoid effectively, which brings pleasure for people in physical and mental.

While compared with the sound quality of the two noise samples before and after adding music, the annoyance level reduced for about 3 levels. In the same site and environment, the original 24 evaluators make the re-evaluation on the sound samples after adding music according to the same division level [9]. The evaluation results are shown in figure 3 .

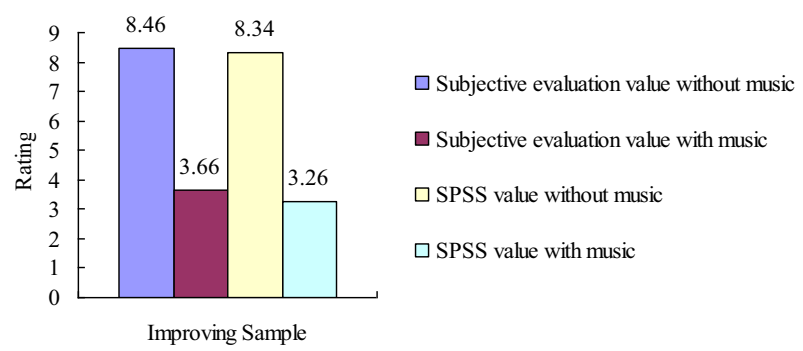

Fig.3. Subjective and objective evaluation value of samples before and after being improved

The subjective evaluation values of annoyance level before and after adding music have made great improvement. The subjective evaluation values of annoyance level increase from 8.46 scores to 3.66 scores, which increase for 9 rankings. At the same time, the consistency of prediction model imitated by SPSS and the values evaluated by the actual listening team is very high. Thus explains using music to improve the vehicle interior sound quality is feasible and effective.

\section{Conclusions}

The effect of 1280 4000 Hz frequency band on the subjective evaluation of the whole frequency band is the largest. The second one is the high frequency band of more than $4000 \mathrm{~Hz}$. The last one is the low frequency band of $20 \sim 160 \mathrm{~Hz}$, which has the smallest effect on the whole frequency band, when regression model is established, the low frequency band can be neglected.

When the samples are under equal loudness process, the samples can be equivalent to a recorded weight function, which will not only make the loudness of each sample be coincident, but also do not change the difference relationship of the remaining variables.

The essence of using music to improve the vehicle interior noise is to raise the tone of vehicle interior noise and reduce the low-pitched degree, so as to reduce the annoyance level and improve the vehicle interior sound quality.

\section{Acknowledgements}

This work was financially supported by both National Natural Science Foundation of China (51575238) and the Jiangsu Colleges and Universities Natural Science Research Project (15KJB580001).

\section{References}

1. X.P. Chen, Acoustics and Psychoacoustics(Posts \& Telecom, Peiking, 2010)

2. Y. Pan, Z.H. Meng, ACTA ACUSTICA 38, 2(2013)

3. D.X. Mao, Y. Wang, Techl Acous 25,6 (2006)

4. Y.H. Gao, Pure Electric Vehicle Interior Sound Quality Analysis and Evaluation System(JLU, Jilin, 2013)

5. J. Zhang, D.X. Mao, Sci. China 40,4 (2010)

6. D.X. Mao, W.Z. Yu and Z.M. Wang, ACTA ACUSTICA 30 (2005)

7. J.H. Yoon, I.H. Yang, J.E. Jeong, App. Acous 73(2012)

8. M.X. Shen, S.G. Zuo, Tech Acous 28,3 (2009)

9. H.Y. Liu, J.P. Gu, Z.H. Meng, Tech Acous 27, 5(2008) 